\title{
AMENABILITY AND DERIVATIONS OF THE FOURIER ALGEBRA
}

\author{
BRIAN FORREST
}

(Communicated by Richard R. Goldberg)

\begin{abstract}
It is shown that a locally compact group $G$ is amenable if and only if every derivation of the Fourier algebra $A(G)$ into a Banach $A(G)$ bimodule is continuous. Also given are necessary and sufficient conditions for $A(G)$ to be weakly amenable.
\end{abstract}

Introduction. If $G$ is an abelian locally compact group, then it is known that every derivation of $A(G)$, the Fourier algebra of $G$, into a Banach $A(G)$-bimodule is continuous $[8$, p. 410]. We shall prove in the first part of this paper that this automatic continuity property for derivations of $A(G)$ characterizes amenable locally compact groups.

In the second part of this paper we will examine the derivations of $A(G)$ into commutative Banach $A(G)$-bimodules. We shall focus our attention on the nature of $V N(G)$, the dual of $A(G)$, as a commutative Banach $A(G)$-bimodule and, in particular, on the $A(G)$-submodule $U C B(\hat{G})$ of $V N(G)$.

$U C B(\hat{G})$ is the norm closure in $V N(G)$ of the linear span of $A(G) \cdot V N(G)$. The definition of $U C B(\hat{G})$ is due to E. Granirer, who studied its properties in [5]. Recently, Bade, Curtis, and Dales [1] introduced the notion of a weakly amenable Banach algebra $\mathscr{A}$ as one in which every continuous derivation of $\mathscr{A}$ into a commutative Banach $\mathscr{A}$-bimodule is zero. We will prove that $A(G)$ is weakly amenable if and only if every continuous derivation of $A(G)$ into $U C B(\hat{G})$ is zero. We also present evidence to suggest that $A(G)$ may well be weakly amenable for a large class of locally compact groups. In particular, if $G$ is discrete, then $A(G)$ is weakly amenable.

This paper will form part of the author's Ph.D. thesis, written under the supervision of Professor Anthony T. Lau. The author would like to express his deep gratitude to Professor Lau, as well as to the Natural Science and Engineering Research Council of Canada and the Alberta Heritage Scholarship Fund for their financial support.

Definitions and notation. Let $G$ be a locally compact group with a fixed left Haar measure $d x$. For each complex-valued function $f$ on $G$ and every $x \in G$ define $L_{x} f(y)=f\left(x^{-1} y\right), f^{\vee}(x)=f\left(x^{-1}\right)$, and $\tilde{f}(x)=\overline{f\left(x^{-1}\right)}$.

$G$ is said to be amenable if there exists $m \in L^{\infty}(G)^{*}$ such that $m \geq 0, m\left(1_{G}\right)=1$ and $m\left(L_{x} f\right)=m(f)$ for every $x \in G, f \in L^{\infty}(G)$.

Received by the editors September 29, 1987.

1980 Mathematics Subject Classification (1985 Revision). Primary 43A07, 43A15; Secondary $46 \mathrm{~J} 10$.

Key words and phrases. Amenable group, weakly amenable, Fourier algebra, derivation. 
The Fourier algebra of $G$, denoted by $A(G)$, is the linear subspace of $C_{0}(G)$ (the continuous complex-valued functions on $G$ which vanish at infinity) consisting of all functions $(f * \tilde{g})^{\vee}$, where $f, g \in L^{2}(G) . V N(G)$ is the closure in the weak operator topology of the linear span of $\left\{L_{x} ; x \in G\right\}$ in $B\left(L^{2}(G)\right)$, the algebra of bounded linear operators on $L^{2}(G) . A(G)$ is the unique predual of the von Neumann algebra $V N(G)$ [3, pp. 210 and 218]. With pointwise multiplication and $\|u\|_{A(G)}=\sup \{|\langle T, u\rangle| ; T \in V N(G),\|T\| \leq 1\}, A(G)$ is a Banach algebra with spectrum $\Delta(A(G))=G[3$, p. 222].

If $I$ is an ideal of $A(G)$, let $Z(I)=\{x \in G ; u(x)=0$ for every $u \in I\}$. If $A \subset G$ is closed, then $I(A)=\{u \in A(G) ; u(x)=0$ for every $x \in A\}$ is a closed ideal of $A(G) . A$ is called a set of spectral synthesis (or simply an $S$-set) if the only closed ideal $I$ in $A(G)$ with $Z(I)=A$ is $I(A)$. An ideal $I$ is called cofinite if $\operatorname{codim} I=\operatorname{dim}(A(G) / I)<\infty$.

An algebraic bimodule $X$ of the Banach algebra $\mathscr{A}$ is called a Banach $\mathscr{A}$ bimodule if $X$ is a Banach space, $\|u \cdot x\| \leq\|u\|\|x\|$ and $\|x \cdot u\| \leq\|u\|\|x\|$ for every $u \in \mathscr{A}, x \in X . X$ is commutative if $x \cdot u=u \cdot x$ for every $u \in \mathscr{A}, x \in X$.

$V N(G)$ becomes a commutative Banach $A(G)$-bimodule by means of the formula $\langle u \cdot T, v\rangle=\langle T \cdot u, v\rangle=\langle T, u v\rangle$ for every $T \in V N(G), u, v \in A(G)$. If $T \in V N(G)$, then $\operatorname{supp} T=\{x \in G ; u(x)=0$ for every $u \in A(G)$ with $u \cdot T=0\}$. A maṕ $\Gamma: V N(G) \rightarrow V N(G)$ is said to be invariant if $u \cdot \Gamma(T)=\Gamma(u \cdot T)$ for every $u \in A(G), T \in V N(G)$.

$P(G)$ denotes the continuous positive definite functions on $G$.

Continuity of derivations on $A(G)$.

DEFINITION 1. Let $\mathscr{A}$ be a Banach algebra and let $X$ be a Banach $\mathscr{A}$-bimodule. A derivation $D: \mathscr{A} \rightarrow X$ is a linear map which satisfies

$$
D(u v)=u \cdot D(v)+D(u) \cdot v \quad \text { for every } u, v \in \mathscr{A} .
$$

LEMMA 1. Let $G$ be a nonamenable locally compact group. Then there exists a discontinuous derivation of $A(G)$ into a finite-dimensional commutative Banach $A(G)$-bimodule.

Proof. If $G$ is nonamenable, then $I^{2}(\{e\})=\left\{\sum_{i=1}^{n} u_{i} v_{i} ; u_{i}, v_{i} \in I(\{e\})\right.$ is not closed in $A(G)\left[4\right.$, Lemma 6.7]. Therefore, her $I^{2}(\{e\})$ is not cofinite or $I^{2}(\{e\})$ is cofinite but not closed. In either case, there exists a nonclosed cofinite ideal $I$ of $A(G)$ such that $I^{2}(\{e\}) \subseteq I \subset I(\{e\})$. Then, as is shown in [2, p. 402], the finite-dimensional space $I(\{e\}) / I$ can be made into a commutative Banach $A(G)$-bimodule and a discontinuous derivation $D: A(G) \rightarrow I(\{e\}) / I$ can be constructed.

LEMMA 2. Let $G$ be an amenable locally compact group. Let $I$ be a closed ideal in $A(G)$ with infinite codimension. Then there exist sequences $\left\{u_{n}\right\},\left\{v_{n}\right\}$ in $A(G)$ such that $u_{n} v_{1} \cdots v_{n-1} \notin I$ but $u_{n} v_{1} \cdots v_{n} \in I$ for all $n \geq 2$.

Proof. Let $A=Z(I)$. Since $G$ is amenable and $I$ has infinite codimension, $A$ is infinite [4, Corollary 5.6].

Assume that $x$ is a cluster point of $A$. Let $x_{1} \in A, x_{1} \neq x$. Let $V_{1}$ be a compact neighborhood of $x_{1}$ with $x \notin V_{1}$. Choose $x_{2} \in A \backslash V_{1}, x_{2} \neq x$. Let $V_{2}$ be a compact neighborhood of $x_{2}$ such that $x \notin V_{2}$ and $V_{1} \cap V_{2}=\varnothing$. Proceeding inductively, 
we get $x_{n} \in A \backslash \bigcup_{i=1}^{n-1} V_{i}, x_{n} \neq x$ and a compact neighborhood $V_{n}$ of $x_{n}$ such that $x \notin V_{n}$ and $V_{n} \cap\left(\bigcup_{i=1}^{n-1} V_{i}\right)=\varnothing$.

If $A$ has no cluster points, then there exists a discrete set $\left\{x_{1}, x_{2}, \ldots\right\} \subseteq A$. Let $V_{1}$ be a compact neighborhood of $x_{1}$ with $\left\{x_{2}, x_{3}, \ldots\right\} \cap V_{1}=\varnothing$. With $V_{n-1}$ chosen, let $V_{n}$ be a compact neighborhood of $x_{n}$ such that $V_{n} \cap\left\{x_{n+1}, x_{n+2}, \ldots\right\}=\varnothing$ and $V_{n} \cap\left(\bigcup_{i=1}^{n-1} V_{i}\right)=\varnothing$.

In either case, we get a sequence $\left\{V_{n}\right\}$ of compact neighborhoods of points $\left\{x_{n}\right\}$ in $A$ such that $V_{n} \cap\left(\bigcup_{i=1}^{n-1} V_{i}\right)=\varnothing$ for $n \geq 2$.

For each $n=1,2, \ldots$, let $u_{n}$ be a function $A(G)$ with $u_{n} \geq 0, \operatorname{supp} u_{n} \subseteq V_{n}$, $u_{n}\left(x_{n}\right)>0$ and $\left\|u_{n}\right\|_{A(G)}=1 / 2^{n}$.

For $k=1,2, \ldots$ define

$$
v_{k}=\sum_{i=k+1}^{\infty} u_{i} .
$$

Since $\sum_{k=1}^{\infty}\left\|u_{k}\right\|_{A(G)}<\infty$, each $v_{k} \in A(G)$.

If $n \geq 2$, then

$$
u_{n} v_{1} \cdots v_{n-1}\left(x_{n}\right)>0 \text {. }
$$

Hence $u_{n} v_{1} \ldots v_{n-1} \notin I$. However, supp $u_{n} \subseteq V_{n}$ and $v_{n}\left(V_{n}\right)=0$. Therefore,

$$
u_{n} v_{1} \cdots v_{n}=0 \in I \text {. }
$$

THEOREM 1. Let $G$ be a locally compact group. Then the following are equivalent:

(i) $G$ is amenable.

(ii) Every derivation of $A(G)$ into a finite-dimensional commutative Banach $A(G)$-bimodule is continuous.

(iii) Every derivation of $A(G)$ into a Banach $A(G)$-bimodule is continuous.

ProOF. That (iii) implies (ii) is trivial. That (ii) implies (i) follows immediately from Lemma 1.

Assume that $G$ is amenable. Let $I$ be a closed cofinite in $A(G)$. By [4, Corollary 6.6], $I^{2}=I$. This, together with Lemma 2 and [6, Theorem 2], implies that every derivation from $A(G)$ into a Banach $A(G)$-bimodule is continuous. That is, (i) implies (iii).

\section{Weak amenability of $A(G)$.}

DEFINITION 2. Let $\varphi$ be a multiplicative linear functional on a Banach algebra $\mathscr{A}$. A point derivation at $\varphi$ is a linear function $d: \mathscr{A} \rightarrow \mathrm{C}$ such that

$$
d(u v)=\varphi(u) d(v)+\varphi(v) d(u) \quad \text { for every } u, v \in \mathscr{A} \text {. }
$$

PROPOSITION 1. Let $G$ be a locally compact group. Then $A(G)$ has no continuous nonzero point derivations at any point in the spectrum of $A(G)$.

Proof. Let $x \in G=\Delta(A(G))$; cf. [3, p. 229]. Let $d$ be a continuous point derivation at $x$. Let $v \in A(G)$ be such that $v(x)=1$ and $\|v\|_{A(G)}=1$. Then

$$
d\left(v^{n}\right)=n d(v) \text { for } n=1,2, \ldots
$$

Since $d$ is bounded, $d(v)=0$. Let $v_{1}, v_{2} \in I(\{x\})$. Then

$$
d\left(v_{1} v_{2}\right)=v_{1}(x) d\left(v_{2}\right)+v_{2}(x) d\left(v_{1}\right)=0 .
$$


$I^{2}(\{x\})$ is an ideal in $A(G)$ with $Z\left(I^{2}(\{x\})\right)=\{x\}$. As $\{x\}$ is an $S$-set [3, p. 229], $I^{2}(\{x\})$ is dense in $I(\{x\})$. Therefore

$$
d(u)=0 \quad \text { for every } u \in I(\{x\}) .
$$

However, if $u \in A(G)$, then $u=u(x) v+(u-u(x) v)$ and

$$
d(u)=u(x) d(v)+d(u-u(x) v)=0 .
$$

LEMMA 3. Let $G$ be a locally compact group. Let $x \in G$. Then there exists a continuous invariant projection of $V N(G)$ onto $\left\langle L_{x}\right\rangle=\left\{\lambda L_{x} ; \lambda \in \mathbb{C}\right\}$.

ProOF. We follow an idea of P. Renaud [7]. Let $\left\{V_{\alpha}\right\}_{\alpha \in \mathfrak{A}}$ be a neighborhood basis at $e$. Let $u_{\alpha} \in P(G) \cap A(G)$ be such that $u_{\alpha}(e)=1$ and $\operatorname{supp} u_{\alpha} \subseteq V_{\alpha}$. Let $m \in V N(G)^{*}$ be a weak-* cluster point of $\left\{L_{x} u_{\alpha}\right\}$. Then, as in [7, Proposition 3 and Theorem 4],

$$
m(u \cdot T)=u(x) m(T) \quad \text { for every } u \in A(G), T \in V N(G) .
$$

Furthermore, $m\left(L_{x}\right)=1$. Therefore, define

$$
P(T)=m(T) L_{x} .
$$

$P$ is an invariant projection of $V N(G)$ onto $\left\langle L_{x}\right\rangle$.

Lemma 4. Let $D: A(G) \rightarrow V N(G)$ be a continuous derivation. Let $X \in G$ and let $P_{x}$ be a continuous invariant projection of $V N(G)$ onto $\left\langle L_{x}\right\rangle$. Define

$$
D_{P_{x}}(u)=P_{x}(D(u)) \quad \text { for every } u \in A(G) \text {. }
$$

Then $D_{P_{x}}$ is a continuous point derivation at $x$. In particular, $D_{P_{x}}=0$.

Proof. Let $u, v \in A(G)$. Then

$$
\begin{aligned}
D_{P_{x}}(u v) & =P_{x}(D(u v))=P_{x}(u \cdot D(v)+v \cdot D(u)) \\
& =u \cdot P_{x}(D(v))+v \cdot P_{x}(D(u)) \\
& =u(x) D_{P_{x}}(v)+v(x) D_{P_{x}}(u) .
\end{aligned}
$$

Let $X$ be a commutative Banach $A(G)$-bimodule and let $D: A(G) \rightarrow X$ be a continuous derivation. For each $\varphi \in X^{*}$ and $u \in A(G)$ define $T_{\varphi, u} \in V N(G)$ by

$$
\left\langle T_{\varphi, u}, V\right\rangle=\varphi(v \cdot D(u)) \text { for every } v \in A(G) .
$$

The main idea in the next lemma is due to Bade, Curtis, and Dales [1].

LEMMA 5. Let $X$ be a commutative Banach $A(G)$-bimodule and let $D: A(G) \rightarrow$ $X$ be a continuous derivation. For each $\varphi \in X^{*}$ and $u \in A(G)$, define $\tilde{D}_{\varphi}(u)=$ $T_{\varphi, u}$. Then $\tilde{D}_{\varphi}: A(G) \rightarrow V N(G)$ is a continuous derivation. Furthermore, if $D$ is nonzero, then for some $\varphi \in X^{*}, \tilde{D}_{\varphi}$ is nonzero.

ProOF. Let $u, v, w \in A(G)$.

$$
\begin{aligned}
(\tilde{D} \varphi(u v))(w) & =\varphi(w \cdot D(u v))=\varphi(w \cdot u \cdot D(v)+w \cdot v \cdot D(u)) \\
& =(\tilde{D} \varphi(v))(u w)+(\tilde{D} \varphi(u))(v w) \\
& =[u \cdot \tilde{D} \varphi(v)](w)+[v \cdot \tilde{D} \varphi(u)](w) .
\end{aligned}
$$

Hence $\tilde{D} \varphi$ is a derivation. Since $\varphi \in X^{*}$ and $D$ is continuous, $\tilde{D} \varphi$ is also continuous. 
As $A(G)^{2}$ is dense in $A(G)[3$, p. 223], if $D$ is nonzero, there exist $u, v \in A(G)$ such that $D(u v) \neq 0$. However,

$$
u v=\frac{(u+v)^{2}-u^{2}-v^{2}}{2}
$$

so we may assume that $D\left(u^{2}\right) \neq 0$ for some $u \in A(G)$. Let $\varphi \in X^{*}$ be such that $\varphi\left(D\left(u^{2}\right)\right) \neq 0$. Then

$$
[\tilde{D} \varphi(u)](u)=\varphi(u \cdot D(u))=\frac{1}{2} \varphi\left(D\left(u^{2}\right)\right) \neq 0 .
$$

LEMMA 6. Let $D: A(G) \rightarrow V N(G)$ be a continuous derivation. Then $D(u) \in$ $U C B(\hat{G})$ for every $u \in A(G)$.

Proof. Let $u, v \in A(G)$. Then $D(u v)=u D(v)+v D(u) \in U C B(\hat{G})$. Since $A^{2}(G)$ is dense in $A(G)$ and $U C B(\hat{G})$ is closed, $D(u) \in U C B(\hat{G})$ for every $u \in$ $A(G)$.

THEOREM 2. Let $G$ be a locally compact group. Then the following are equivalent:

(i) $A(G)$ is weakly amenable.

(ii) Every continuous derivation from $A(G)$ into $U C B(\hat{G})$ is zero.

Proof. That (i) implies (ii) is trivial.

Conversely, it follows from Lemma 5 and Lemma 6 that if $A(G)$ is not weakly amenable, then there exists a nonzero continuous derivation of $A(G)$ into $U C B(\hat{G})$.

Theorem 2 is a stronger version of [1, Theorem 1.5].

Proposition 2. Let $D: A(G) \rightarrow U C B(\hat{G})$ be a continuous derivation and let $u \in A(G)$. Then $\operatorname{supp} D(u)$ contains no isolated points.

ProOF. Assume that $V$ is an open neighborhood of $x \in \operatorname{supp} D(u)$ with $V \cap$ $\operatorname{supp} D(u)=\{x\}$. Let $v \in A(G)$ be such that $v(x)=1$ and $\operatorname{supp} v \subseteq V$. Define $D_{0}: A(G) \rightarrow U C B(\hat{G})$ by $D_{0}(w)=v \cdot D(w)$ for every $w \in A(G)$. Then $D_{0}$ is a continuous derivation. As $v(x)=1, x \in \operatorname{supp} D_{0}(u)[3$, p. 225]. But $\operatorname{supp} v \cdot D(u) \subseteq$ $\operatorname{supp} v \cap \operatorname{supp} D(u)=\{x\}$. Therefore $\operatorname{supp} D_{0}(u)=\{x\}$ and $D_{0}(u)=\lambda L_{x}$ for some $\lambda \neq 0[3$, p. 229].

By lemma 3, there exists a continuous invariant projection $P_{x}$ of $V N(G)$ onto $\left\langle L_{x}\right\rangle$. By lemma $4, P_{x} \circ D_{0}$ is a nonzero continuous point derivation of $A(G)$ at $x$, contradicting Proposition 1.

THEOREM 3. Let $G$ be a discrete group. Then $A(G)$ is weakly amenable.

PROOF. This follows immediately from Theorem 2 and Proposition 2 if we note that $\operatorname{supp} T=\varnothing$ if and only if $T=0$ [3, p. 224].

THEOREM 4. Let $G$ be a discrete group. Then the following are equivalent:

(i) $G$ is amenable.

(ii) Every derivation from $A(G)$ into a finite-dimensional commutative Banach $A(G)$-bimodule is zero.

Proof. If $G$ is amenable, then every derivation is continuous (Theorem 1). Therefore (ii) holds by Theorem 3. If $G$ is nonamenable, then by Lemma 1, there 
exists a discontinuous derivation of $A(G)$ into a commutative finite-dimensional Banach $A(G)$-bimodule.

It seems that $A(G)$ is weakly amenable for every locally compact group $G$ (or at least for all amenable locally compact groups). We feel that the key to the proof of this statement lies in a stronger version of Proposition 2.

\section{REFERENCES}

1. W. G. Bade, P. C. Curtis and H. Dales, Amenability and weak amenability for Beurling and Lipschitz algebras (preprint).

2. H. Dales and G. Willis, Cofinite ideals in Banach algebras and finite-dimensional representations of group algebras (Proc. Conf. Automatic Continuity...), Lecture Notes in Math., vol. 975, Springer-Verlag, New York, 1982.

3. P. Eymard, L'algèbre de Fourier d'un groupe localement compact, Bull. Soc. Math. France 92 (1964), 181-236.

4. B. Forrest, Amenability and bounded approximate identities in ideals of $A(G)$ (preprint).

5. E. Granirer, Weakly almost periodic and uniformly continuous functionals on the Fourier algebra of any locally compact group, Trans. Amer. Math. Soc. 189 (1974), 371-382.

6. N. P. Jewell, Continuity of module of higher derivations, Pacific J. Math. 68 (1977), 91-98.

7. P. F. Renaud, Invariant means on a class of von Neumann algebras, Trans. Amer. Math. Soc. 170 (1972), 285-291.

8. G. A. Willis, The continuity of derivations from group algebras and factorization in cofinite ideals, (Proc. Conf. Automatic Continuity...), Lecture Notes in Math., vol. 975, Springer-Verlag, New York, 1982.

Department of Mathematics, University of Alberta, Edmonton, Alberta, CANADA

Current address: Department of Mathematics and Statistics, Queen's University, Kingston, Ontario, Canada 